Tohoku J. Exp. Med., 2010, 222, 201-210

\title{
Hypertension Promotes Phosphorylation of Focal Adhesion Kinase and Proline-Rich Tyrosine Kinase 2 in Rats: Implication for the Pathogenesis of Hypertensive Vascular Disease
}

\author{
Koichiro Sugimura, ${ }^{1}$ Yoshihiro Fukumoto, ${ }^{1}$ Jun Nawata, ${ }^{1}$ Huan Wang, ${ }^{1}$ \\ Noriko Onoue, ${ }^{1}$ Tomohiro Tada, ${ }^{1}$ Kunio Shirato ${ }^{1}$ and Hiroaki Shimokawa ${ }^{1}$ \\ ${ }^{1}$ Department of Cardiovascular Medicine, Tohoku University Graduate School of Medicine, Sendai, Japan
}

\begin{abstract}
Atherosclerosis is initiated by adhesion and infiltration of inflammatory leukocytes into the intima, where non-receptor protein tyrosine kinases, such as focal adhesion kinase (FAK) and proline-rich tyrosine kinase 2 (PYK2), play important roles as intracellular messengers of mechanical and biochemical signals. In the present study, we examined whether FAK and PYK2 are up-regulated by elevated blood pressure or circulating humoral factors in hypertension. We used a rat model of abdominal aortic banding that allows separate evaluation of elevated blood pressure (upper body) and circulating humoral factors (lower body). We obtained the proximal and distal aortas of the banding site, 6 hours, 3 days, and 1 and 4 weeks after the banding procedure, for evaluation of phosphorylation of FAK and PYK2 by Western blotting. Arterial pressure was significantly elevated only in the upper body throughout the experimental period. The expression of FAK and the FAK phosphorylation were significantly increased at 1 and 4 weeks only in the proximal aorta. This was also the case for the expression of total PYK2 and the PYK2 phosphorylation. In contrast, there was no significant change in FAK or PYK2 phosphorylation in the distal aorta, whereas plasma levels of angiotensin II were systemically elevated. In sham-operated rats, no change in FAK or PYK2 phoshorylation was noted in the proximal and distal aortas. These results indicate that phosphorylation of FAK and PYK2 is upregulated by elevated blood pressure but not by humoral factors in the rat aorta, demonstrating novel aspects of atherogenesis in hypertension.
\end{abstract}

Keywords: focal adhesion kinase; proline-rich tyrosine kinase 2; integrins, humoral factors; angiotensin II Tohoku J. Exp. Med., 2010, 222 (3), 201-210. 을 2010 Tohoku University Medical Press

Atherosclerosis is the major cause of death in developed countries, which is initiated by adhesion and infiltration of inflammatory leukocytes into the intima (Ross 1999). In the process of atherosclerosis, inflammatory cytokines and adhesion molecules play important roles to develop atheroma that consists of fibrous cap and lipid core (Fukumoto et al. 1997; Ross 1999; Shimokawa 2000; Libby and Aikawa 2002; Fukumoto et al. 2004). It has been demonstrated that chronic hypertension accelerates vascular smooth muscle cells (VSMC) hypertrophy and extracellular matrix accumulation, which are substantially involved in the development of vascular remodeling and atherosclerosis (Lee et al. 1983; Schwartz et al. 1986; Bezie et al. 1998). Vascular remodeling induced by hypertension is mainly caused by two factors; increased mechanical stress and up-regulated humoral factors such as angiotensin II (Tamura et al. 1998; Shimokawa 2002). However, it remains to be examined to which extent these two mechanisms are involved in vascular remodeling in hypertension.

Recent studies have suggested that non-receptor protein tyrosine kinases play an important role in transducing mechanical stimuli to biochemical pathways by coupling with integrin (Burridge et al. 1992; Fukumoto et al. 1996; Sieg et al. 1998; Schlaepfer et al. 1999; Ostergaard and Lysechko 2005; Ramjaun and Hodivala-Dilke 2009). Among them, focal adhesion kinase (FAK) is a non-receptor protein tyrosine kinase that localizes to the point of cell contact with extracellular matrix and has been demonstrated to activate mitogen-activated protein kinases (MAPK) in response to integrin clustering in various types of cells (Burridge et al. 1992; Sieg et al. 1998; Schlaepfer et al. 1999; Ostergaard and Lysechko 2005; Vadali et al. 2007; Cai et al. 2008). Proline-rich tyrosine kinase 2 (PYK2) is another non-receptor protein tyrosine kinase that has a high sequence homology to FAK, sharing $45 \%$ amino-acid sequence identity (Sieg et al. 1998; Avraham et al. 2000;

Received October 4, 2010; revision accepted for publication October 19, 2010. doi: 10.1620/tjem.222.201

Correspondence: Yoshihiro Fukumoto, M.D., Ph.D., Department of Cardiovascular Medicine, Tohoku University Graduate School of

Medicine, 1-1, Seiryo-Machi, Aoba-ku, Sendai 980-8574, Japan.

e-mail: fukumoto@cardio.med.tohoku.ac.jp 
Rocic et al. 2002). In FAK-null cells, PYK2 expression is increased, and PYK2 phosphorylation by fibronectin activates extracellular signal-related kinases (ERK) (Sieg et al. 1998). Phosphorylation of these kinases is accelerated by mechanical stress (Rice et al. 2002; Iwasaki et al. 2003). However, FAK and PYK2 could also be activated by angiotensin II, contributing to vascular remodeling in hypertension (Govindarajan et al. 2000; Rocic et al. 2002).

In the present study, we thus examined whether FAK and PYK2 are activated in hypertension and if so, to which extent elevated blood pressure and up-regulated humoral factors (e.g. angiotensin II) are involved. In order to address this important issue, we used a rat model of abdominal aortic banding, which allows separate evaluation of elevated blood pressure (upper body alone) and up-regulated humoral factors (whole body).

\section{Methods}

All procedures were performed according to the protocols approved by the Institutional Committee for Use and Care of Laboratory Animals of Tohoku University and the Guide for Care and Use of Laboratory Animals published by the U.S. National Institutes of Health (NIH Publication No. 85-23, revised 1996).

\section{Animal Preparation}

Eighty male Wistar rats initially weighing $180-220 \mathrm{~g}$ were anesthetized with intraperitoneal pentobarbital sodium $(50 \mathrm{mg} / \mathrm{kg})$. The rat abdominal aorta banding was performed, as previously described (Wang et al. 2004). Their abdominal aortas proximal to the bilateral renal arteries were tightly tied with a $0.8 \mathrm{~mm}(21 \mathrm{G})$ external diameter needle, and then the needle was removed, leaving a constricted aorta (AO group, $n=10$ each for 6 hours, 3 days, 1 week and 4 weeks, respectively). Sham-operated rats served as controls (SO group, $n=$ 10 for each group). All rats were housed in wire cages and fed a standard rodent chow and tap water ad libitum. Six hours, 3 days, 1 and 4 weeks after the operation, right cervical and femoral arterial pressures were measured under pentobarbital anesthesia. Then, blood samples were collected to examine circulating plasma renin activity and angiotensin II by radioimmunoassay, as previously described (Wang et al. 2004). At the end of blood sampling, the aortas were rapidly removed and separated the proximal and distal aortas from the banding site.
The tissues were directly frozen in liquid nitrogen and stored at $-80^{\circ} \mathrm{C}$ until the use for Western blot.

\section{Reagents}

We used mouse anti-human FAK monoclonal antibody (clone 4.47), rabbit anti-human FAK phosphospecific polyclonal antibody (pTyr397), mouse anti-human PYK2 monoclonal antibody (clone 74), mouse anti-human PYK2 phosphospecific monoclonal antibody (pTyr402, clone RR102, cross-reactive with rat) (all from BD Pharmingen, San Diego, CA, USA). We also used rabbit anti-human integrin $\beta-1$ (M-106) and goat anti-human integrin $\beta-3$ (N-20) polyclonal antibody (Sigma, St. Louis, MO, USA) as primary antibodies. We also used alkaline phosphatase-conjugated goat anti-mouse secondary antibody (American Qualex, San Clemente, CA, USA), alkaline phosphatase-conjugated swine anti-rabbit secondary antibody (Dako, Glostrup, Denmark), and alkaline phosphatase-conjugated rabbit anti-goat secondary antibody (Sigma, St. Louis, MO, USA) (Nakano et al. 2007; Saji et al. 2007; Do e et al. 2009).

\section{Western Blot Analysis}

FAK phosphorylation is necessary for activation of downstream signaling cascades leading to cytoskeletal reorganization and cellular growth (Schlaepfer and Hunter 1997; Schlaepfer et al. 1999). Thus, we examined the extent of FAK phosphorylation at tyrosine residue 397, one of the major phosphorylation sites of FAK that leads to MAPK activation of MAPK (Schlaepfer and Hunter 1997). Similarly, we examined the extent of PYK2 phosphorylation at tyrosine residue 402, the most important phosphorylation site of PYK2 that is associated with MAPK activation (Avraham et al. 2000). Integrins are mechanosensors that perceive changes in extracellular forces and act as molecular switches leading to downstream signaling through FAK to regulate vascular adaptation in hypertension ( $\mathrm{Li}$ and Xu 2000; Shyy and Chien 2002). Thus, we also examined the expression of $\beta 1$ and $\beta 3$ integrin subunits.

The excised rat aortic tissues were prepared by homogenization in ice-cold buffer containing $10 \mathrm{mmol} / \mathrm{L}$ Tris $\mathrm{pH} 7.4,150 \mathrm{mmol} / \mathrm{L}$ $\mathrm{NaCl}, 1.2 \mathrm{mmol} / \mathrm{L} \mathrm{MgCl}, 1 \mathrm{mmol} / \mathrm{L}$ EGTA, $1 \mathrm{mmol} / \mathrm{L} \mathrm{Na}_{3} \mathrm{VO}_{4}, 10$ $\mathrm{mmol} / \mathrm{L} \mathrm{Na}$ pyrophosphate, $100 \mathrm{mmol} / \mathrm{L} \mathrm{NaF}, 50 \mathrm{mmol} / \mathrm{L}$ HEPES, $\mathrm{pH}$ $7.4,1 \%$ Triton $\mathrm{X}-100,1 \%$ sodium deoxycholate, $10 \mu \mathrm{g} / \mathrm{mL}$ leupeptin, $10 \mathrm{mg} / \mathrm{mL}$ aprotinin, and $1 \mathrm{mmol} / \mathrm{L}$ phenylmethylsulfonyl fluoride. The homogenates were centrifuged at 13,000 $\mathrm{g}$ for $5 \mathrm{~min}$ and the supernatants were collected. The protein contents were measured

Table 1. Effect of abdominal aortic banding on body weight, heart weight and blood pressure.

\begin{tabular}{|c|c|c|c|c|c|c|c|c|}
\hline & \multicolumn{2}{|c|}{6 hours } & \multicolumn{2}{|c|}{3 days } & \multicolumn{2}{|c|}{1 week } & \multicolumn{2}{|c|}{4 weeks } \\
\hline & Sham & $\begin{array}{c}\text { Aortic } \\
\text { banding }\end{array}$ & Sham & $\begin{array}{c}\text { Aortic } \\
\text { banding }\end{array}$ & Sham & $\begin{array}{c}\text { Aortic } \\
\text { banding }\end{array}$ & Sham & $\begin{array}{c}\text { Aortic } \\
\text { banding }\end{array}$ \\
\hline$n$ & 10 & 10 & 10 & 10 & 10 & 10 & 10 & 10 \\
\hline BW (g) & $192 \pm 3$ & $195 \pm 2$ & $225 \pm 4$ & $198 \pm 7 *$ & $251 \pm 2$ & $222 \pm 6^{*}$ & $383 \pm 6$ & $362 \pm 4^{*}$ \\
\hline LVW/BW (mg/g) & $2.4 \pm 0.1$ & $2.3 \pm 0.1$ & $2.2 \pm 0.1$ & $2.9 \pm 0.1^{*}$ & $2.3 \pm 0.1$ & $2.8 \pm 0.1 *$ & $2.0 \pm 0.1$ & $2.7 \pm 0.1^{*}$ \\
\hline Carotid BP $(\mathrm{mm} \mathrm{Hg})$ & $91 \pm 4.4$ & $116 \pm 4.4^{\dagger}$ & $96.0 \pm 2.1$ & $135 \pm 4.9^{* \dagger}$ & $102 \pm 2.7$ & $138 \pm 4.3^{* \dagger}$ & $114 \pm 2.8$ & $146 \pm 3.8^{* \dagger}$ \\
\hline Femoral BP (mm Hg) & $96.0 \pm 2.6$ & $85.0 \pm 5.2$ & $95.0 \pm 0.9$ & $100 \pm 5.6$ & $104 \pm 2.1$ & $105 \pm 3.9$ & $112 \pm 1.1$ & $113 \pm 3.3$ \\
\hline Plasma angiotensin II (pg/ml) & $17.0 \pm 2.5$ & $48.4 \pm 8.7^{*}$ & $16.0 \pm 1.8$ & $70.4 \pm 17.3^{*}$ & $16.0 \pm 4.1$ & $34.3 \pm 6.8^{*}$ & $15.4 \pm 3.6$ & $18.6 \pm 4.1$ \\
\hline Plasma renin activity $(\mathrm{ng} / \mathrm{ml} / \mathrm{h})$ & $15.5 \pm 0.6$ & $34.1 \pm 6.4^{*}$ & $17.3 \pm 1.2$ & $41.6 \pm 5.6^{*}$ & $12.6 \pm 1.7$ & $42.7 \pm 6.0^{*}$ & $11.5 \pm 1.8$ & $9.4 \pm 1.1$ \\
\hline
\end{tabular}

BW, body weight; LVW, left ventricular weight; BP indicates systoric intra-arterial BP. Values are mean \pm S.D. $* P<0.05$ vs sham; ${ }^{\dagger} P<0.05$ vs Femoral BP. 
with a protein assay kit (Bio-Rad Laboratories, CA, USA). Twenty $\mu \mathrm{g}$ of each sample supplemented with $3 \mathrm{X}$ SDS were electrophoresed on a $7.5 \%$ SDS-polyacrylamide gel under a nonreducing condition. The protein on the gel was consequently transferred to the PVDF membrane (Bio-Rad Laboratories, CA, USA). The membrane was incubated over night with the primary antibodies used at a concentration of 1:1,000 (FAK, pY397, PYK2, pY407), 1:100 (integrin $\beta 1$ and $\beta 3)$, followed by the second antibodies conjugating alkaline phosphatase. The membrane was developed with 5-bromo-4-chloro-3-indolyl phosphate and nitro blue tetrazolium. Signals were visualized by the ECL detection system (Amersham Biosciences, Uppsala, Sweden)
(Nakano et al. 2007; Do e et al. 2009).

\section{Statistical Analysis}

Statistical analyses were performed with StatView (StatView 5.0, SAS Institute Inc., Cary, NC). Comparisons of parameters between 2 groups under different conditions were made by 2-way ANOVA, followed by Scheffe's test. All values are presented as mean \pm SEM. A value of $P<0.05$ was considered to be statistically significant.

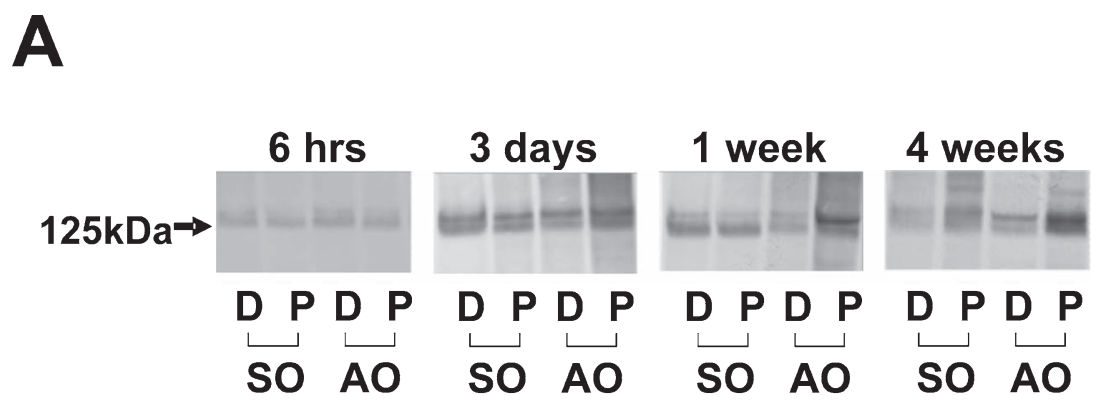

B
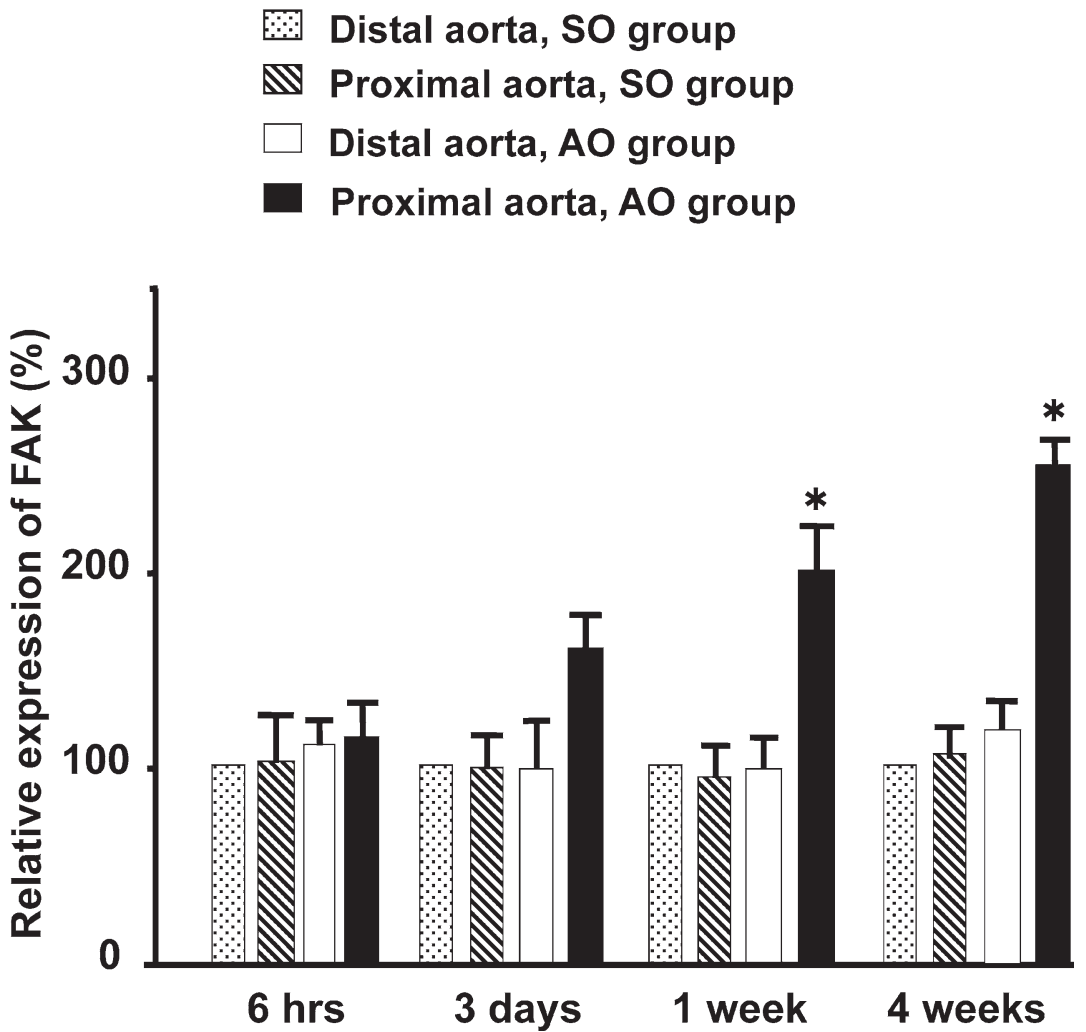

Fig. 1. FAK expression is enhanced by elevated blood pressure.

(A) Representative Western blot showing the expression of FAK in the proximal and distal aortas from the banding site in the aorta-banded (AO) and sham-operated (SO) groups at 6 hours, 3 days, 1 and 4 weeks after the operation. (B) Quantitative analysis of FAK expression. FAK expression was normalized to the level of expression in the distal aorta of the $\mathrm{SO}$ group at each period. Results are expressed as mean \pm SEM. $* P<0.05$ vs. other 3 groups. 
A

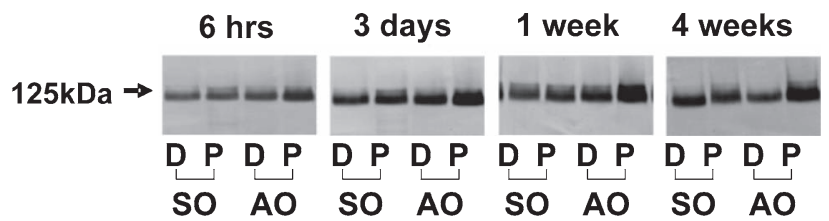

B

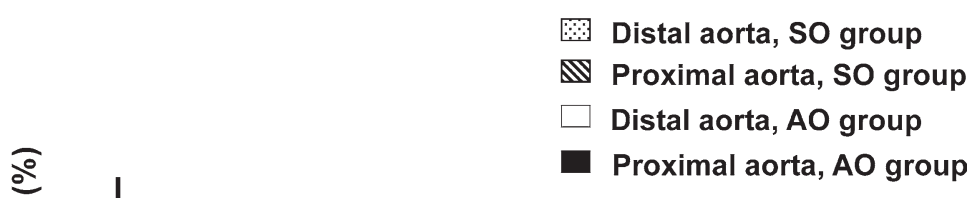

C
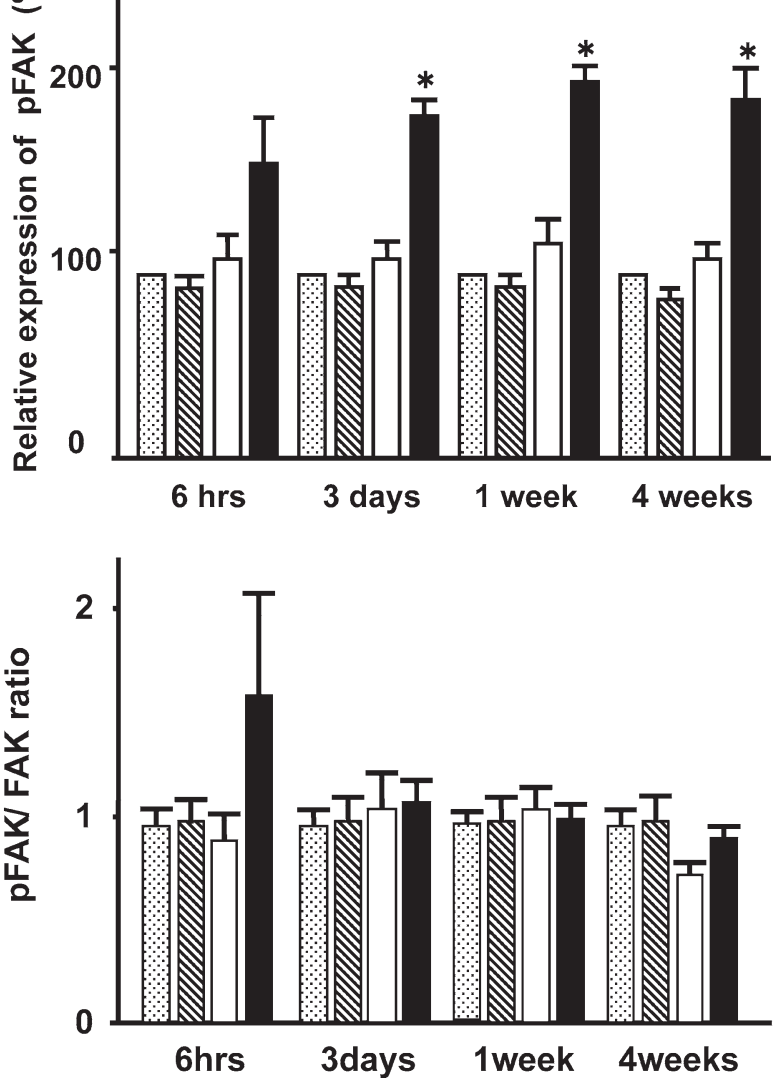

Fig. 2. FAK phosphorylation is enhanced by elevated blood pressure.

(A) Representative Western blot showing the expression of pFAK in the proximal and distal aortas from the banding site in the aorta-banded (AO) and sham-operated (SO) groups at 6 hours, 3 days, and 1 and 4 weeks after the operation. (B) Quantitative analysis of pFAK. pFAK expression was normalized to the level of expression in the distal aortas of the SO group at each period. (C) There was no significant difference in the ratio of FAK phosphorylation to FAK expression. Results are expressed as mean \pm SEM. $* P<0.05$ vs. other 3 groups.

\section{Results}

Blood Pressure and Left Ventricular Hypertrophy

Abdominal aortic banding significantly increased blood pressure in the carotid artery but not in the femoral artery at 6 hours after the procedure, and then maintained the selective hypertensive state in upper body at 3 days and 1 and 4 weeks after the procedure, as compared with the sham-operated group (Table 1). Left ventricular hypertrophy was developed at 3 days after abdominal aorta banding (Table 1). Plasma levels of angiotensin II and renin activity were significantly elevated at 6 hours, 3 days and 1 week in the aorta-banded group as compared with the sham-operated group, whereas these values were reduced at 4 weeks with no difference between the 2 groups (Table 1).

\section{Expression and Phosphorylation of FAK}

Western blotting showed that the expression of FAK (Fig. 1) and FAK phosphorylation at tyrosine residue 397 (Fig. 2A-B) were progressively enhanced only in the proximal aorta but not in the distal aorta in the AO group as compared with the SO group. In contrast, no changes were noted in the SO group. However, there was no significant difference in the ratio of FAK phosphorylation to FAK expression during the experiment (Fig. 2C). 
A

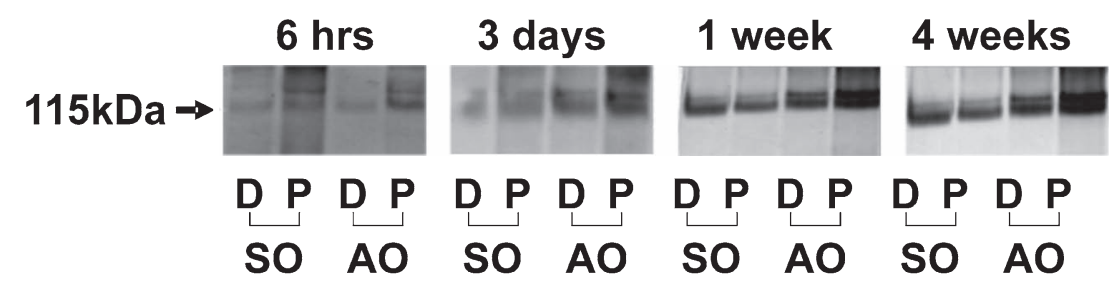

B

Distal aorta, SO group

Proximal aorta, SO group

$\square$ Distal aorta, AO group

- Proximal aorta, AO group

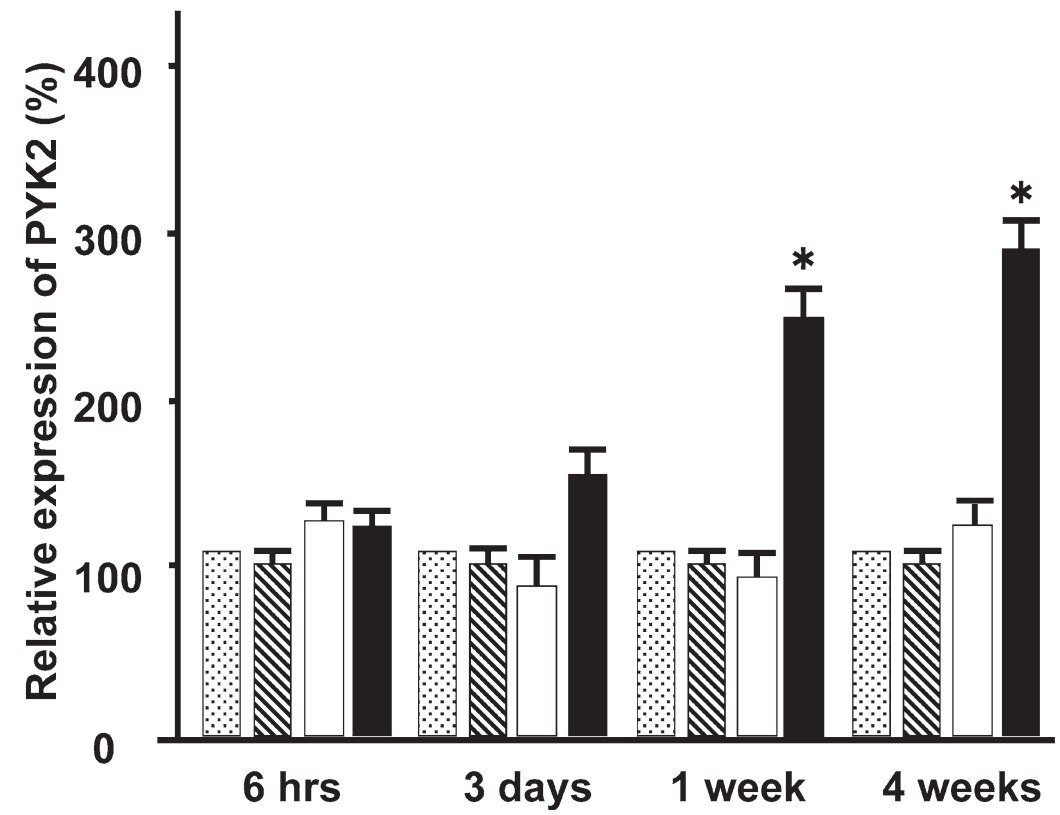

Fig. 3. PYK2 expression is enhanced by elevated blood pressure.

(A) Representative Western blot showing the expression of PYK2 in the proximal and distal aortas from the banding site in the aorta-banded (AO) and sham-operated (SO) groups at 6 hours, 3 days, and 1 and 4 weeks after the operation. (B) Quantitative analysis of PYK2. PYK2 expression was normalized to the level of expression in the distal aortas of the SO group at each period.

Results are expressed as mean \pm SEM. $* P<0.05$ vs. other 3 groups.

Expression and Phosphorylation of PYK2

Western blotting showed that the expression of PYK2 (Fig. 3) and PYK2 phosphorylation at tyrosine residue 402 (Fig. 4A-B) were significantly enhanced at 1 and 4 weeks only in the proximal aorta but not in the distal aorta in the $\mathrm{AO}$ group as compared with the $\mathrm{SO}$ group. In contrast, no changes were noted in the $\mathrm{SO}$ group. However, there was no significant difference in the ratio of PYK2 phosphorylation to PYK2 expression during the experiment (Fig. 4C).

\section{Expression of Integrin $\beta 1$ and $\beta 3$}

Western blotting showed that no changes were noted for integrin $\beta 1$ (Fig. 5) or integrin $\beta 2$ (Fig. 6) in both groups.

\section{Discussion}

The novel finding of the present study is that the expression and phosphorylation of FAK and PYK2 in the aortic wall are enhanced by mechanical stress but not by systemic humoral factors in rats in vivo and that altered 


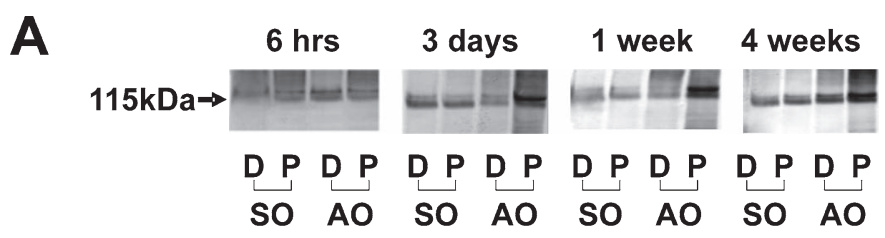

B
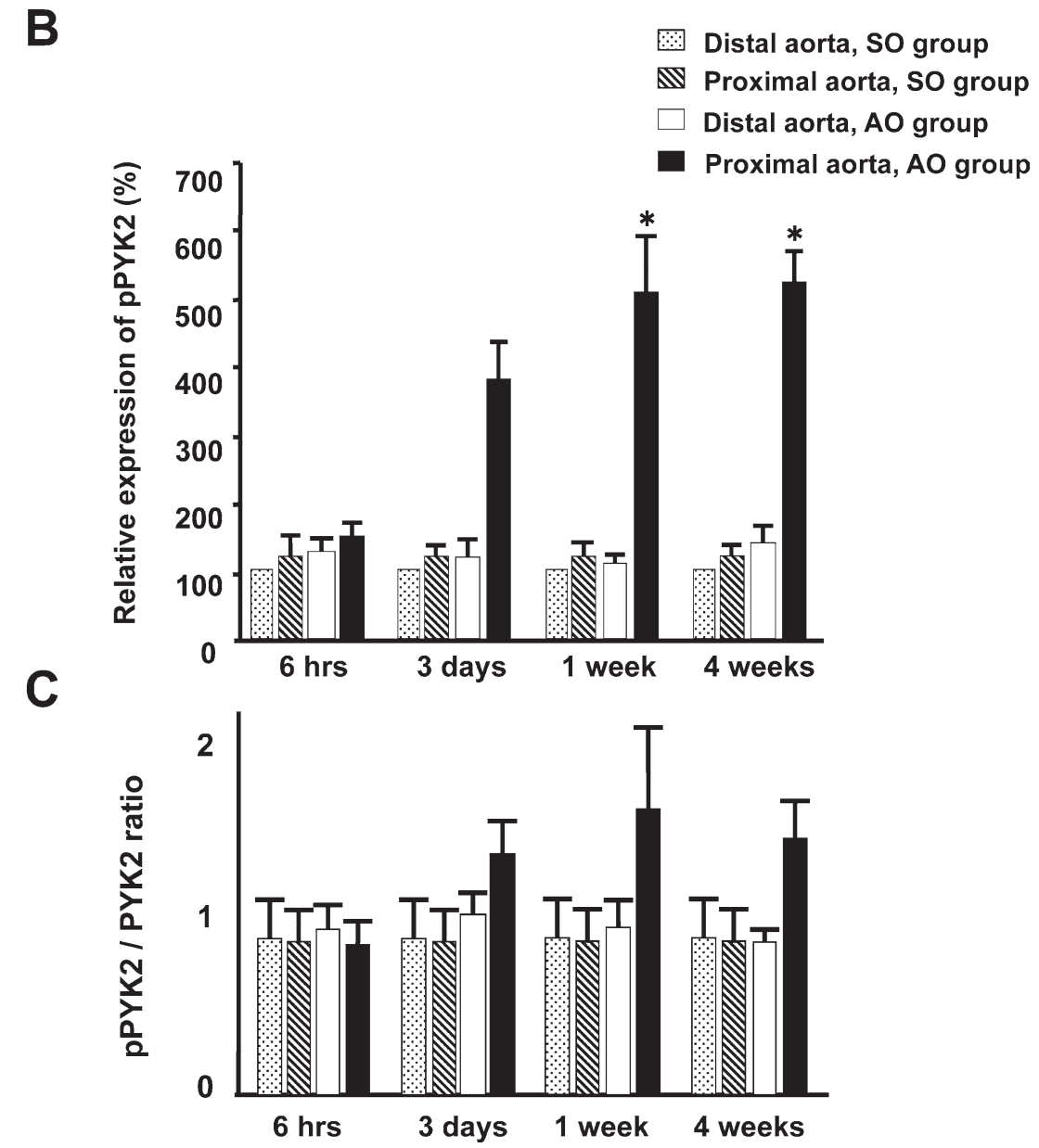

Fig. 4. PYK2 phosphorylation is enhanced by elevated blood pressure.

(A) Representative Western blot showing the expression of pPYK2 in the proximal and distal aortas from the banding site in the aorta-banded (AO) and sham-operated (SO) groups at 6 hours, 3 days, and 1 and 4 weeks after the operation. (B) Quantitative analysis of pPYK2. pPYK2 expression was normalized to the level of expression in the distal aortas of the SO group at each period. (C) There was no significant difference in the ratio of PYK2 phosphorylation to PYK2 expression.

Results are expressed as mean \pm SEM. $* P<0.05$ vs. other 3 groups.

expression of integrins may not be involved in the present rat model of hypertension. Since FAK and PYK2 are the important non-receptor tyrosine kinases that play an important role in transducing mechanical stress to biochemical pathways by coupling with integrins, the present study demonstrates the importance of mechanical stress per se in the atherogenesis of hypertension.

\section{Mechanical Stress and Vascular Remodeling}

In hypertension, vascular remodeling can be induced by both elevated blood pressure and enhanced circulating humoral factors (Lehoux and Tedgui 1998; Fukumoto et al. 2007). It has been shown that the mechanical stress gener- ate intracellular signals through several growth factor receptors, ion-channels, cytoskeletons, all of which are the candidates of sensing mechanical stress and among them, integrins are important mechanosensors (Lehoux and Tedgui 1998; Li and Xu 2000; Ali and Schumacker 2002; Shyy and Chien 2002).

Integrins on the cell surface are the receptors of extracellular matrix that modulate tissue morphology and function (Giancotti and Ruoslahti 1999) by transmitting extracellular signals into cells through activation of integrinassociated protein such as FAK. FAK is known to co-localize the integrin receptors at cell-substratum contact sites termed as focal adhesions and to be phosphorylated by 


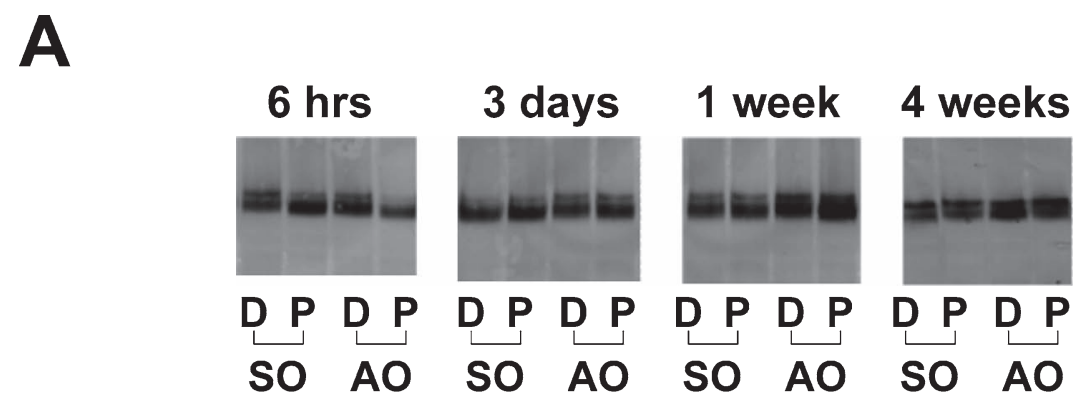

B

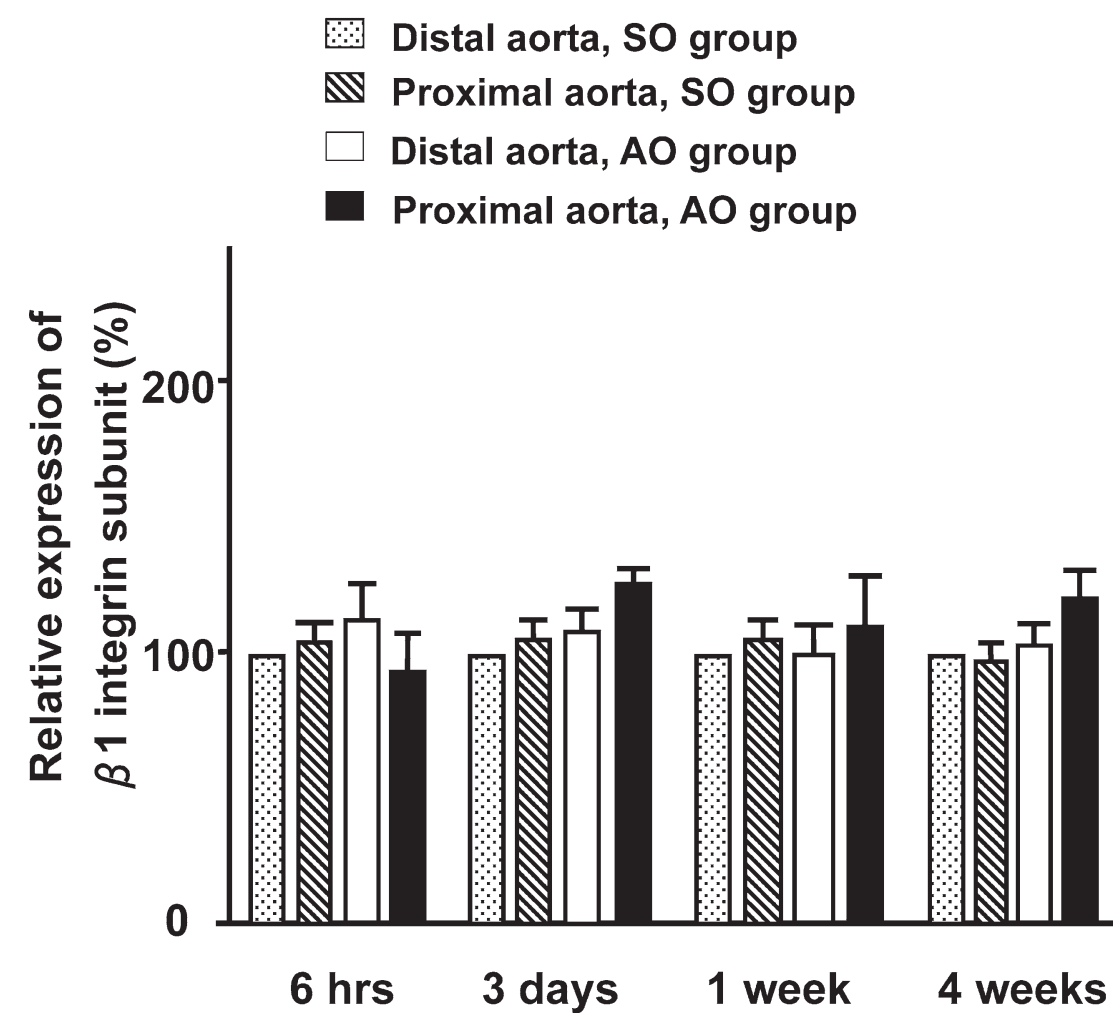

Fig. 5. Expression of $\beta 1$ integrin is unaltered in hypertension.

(A) Representative Western blot showing the expression of $\beta 1$-integrin subunit in the proximal and distal aortas from the banding site in the aorta-banded (AO) and sham-operated (SO) groups. (B) Quantitative analysis of $\beta 1$-integrin. Integrin expression was normalized to the level of expression in the distal aortas of the SO group at each period. Results are expressed as mean \pm SEM. $* P<0.05$ vs. other 3 groups.

clustering of integrins (Schlaepfer et al. 1999). Mechanical stretch promotes FAK phosphorylation in various types of cells followed by activation of MAP kinase cascades ( $\mathrm{Li}$ and Earp 1997; Smith et al. 1998; Torsoni et al. 2003). It has also been reported that FAK phosphorylation is enhanced by pressure stimulus in isolated mesenteric arteries (Rice et al. 2002). Taken together, these results indicate that FAK plays a central role in mechanical signal transduction by coupling with integrins.

Among several sites in the integrin-stimulated FAK tyrosine-phosphorylation, tyrosine residue 397 (Tyr397) is the most important phosphorylated site because as FAK with mutant Tyr397 failed to activate ERK stimulated by fibronectin (Schlaepfer and Hunter 1997). Furthermore, Try402 site of PYK2 is also known to be one of the major phosphorylation sites of PYK2, which plays an important role in the MAP kinase activation through the PYK2 signaling pathway (Avraham et al. 2000). In the present study, we were able to demonstrate that the expression of FAK and PYK2, as well as the phosphorylation of these molecules, 


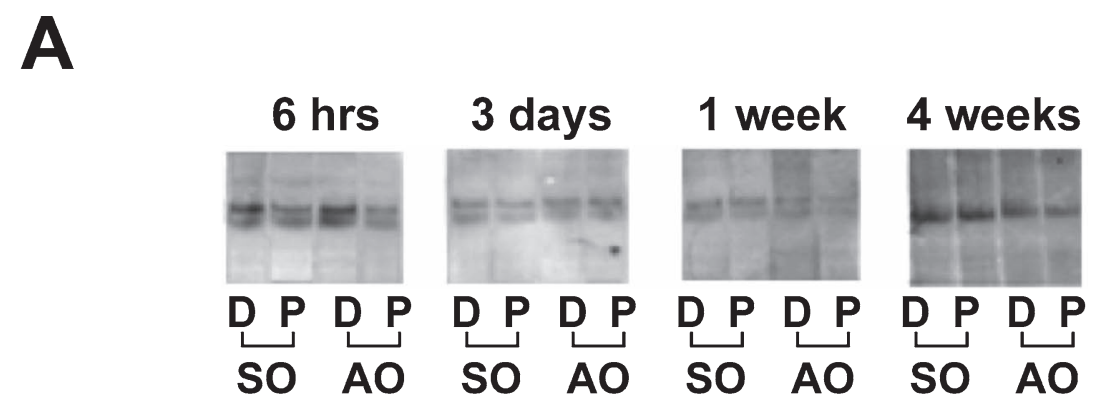

B

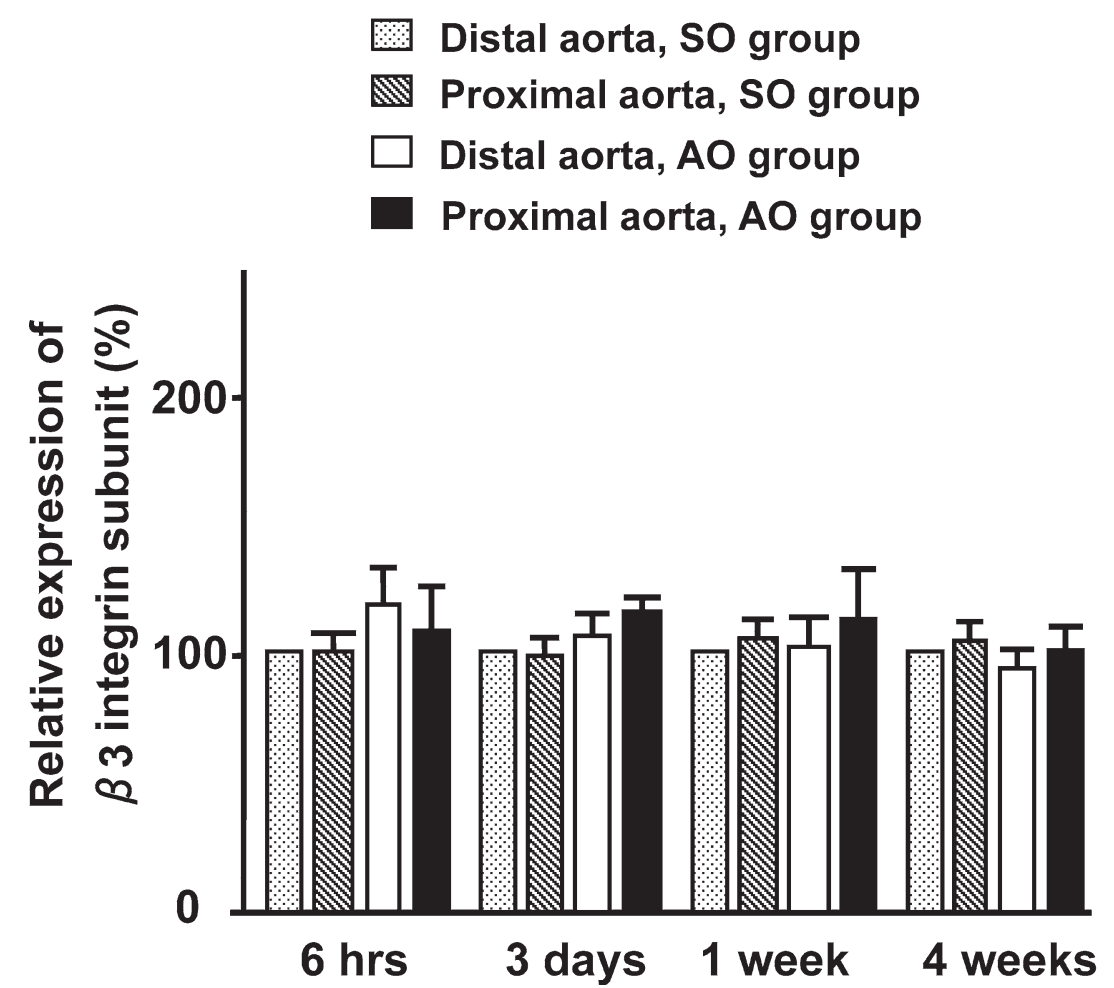

Fig. 6. Expression of $\beta 3$ integrin is unaltered in hypertension.

(A) Representative Western blot showing the expression of $\beta 3$-integrin subunit in the proximal and distal aortas from the banding site in the aorta-banded (AO) and sham-operated (SO) groups. (B) Quantitative analysis of $\beta 3$-integrin. Integrin expression was normalized to the level of expression in the distal aortas of the SO group at each period. Results are expressed as mean \pm SEM. $* P<0.05$ vs. other 3 groups.

were significantly enhanced only in the proximal aorta but not at the distal aorta at 1 and 4 weeks as compared with the sham-operated rats. However, there might be differential roles of FAK and PYK2 signalings in the remodeling of hypertensive rat aorta. Indeed, it has been reported that PYK2 overexpression is associated apoptosis (Xiong and Parsons 1997), whereas FAK overexpression promotes cell survival in vitro (Chan et al. 1999). Thus, it is conceivable that the increased expression and phosphorylation of both
FAK and PYK2 in remodeling of hypertensive aorta results in differential activation of downstream signaling to individual MAP kinase cascade.

\section{Humoral Factors and Vascular Remodeling}

In cultured VSMC, it has been reported that angiotensin II increases phosphorylation of FAK and PYK2 (Govindarajan et al. 2000; Rocic et al. 2002); however, the differential effects of blood pressure and humoral factors 
remain to be elucidated. In the present study, we thus used the rat model of abdominal aorta-banding with the plasma levels of circulating humoral factors but with different blood pressure between the upper and lower bodies and the results indicated that circulating humoral factors may not substantially be involved in the phosphorylation of FAK and PYK2 in the present model.

It was demonstrated that overexpression of FAK protein in cultured fibroblasts leads to activation of extracellular-related kinase (Schlaepfer et al. 1997) and that overexpression of PYK2 protein also accelerates phosphorylation of the downstream Src, Shc, and p130 ${ }^{\mathrm{CAS}}$ (Li and Earp 1997; Sieg et al. 1998). These findings suggest that phosphorylation of tyrosine kinases may be enhanced not only by phosphorylation of the upstream tyrosine kinases but also by that of a total amount of protein expression of those molecules. In the present study, we were able to demonstrate that acute and chronic hypertension enhances the protein expression of FAK and PYK2 as well as the phosphorylation of these molecules in the proximal aorta of aorta-banded rats.

\section{Limitations of the Study}

Several limitations should be mentioned for the present study. First, we have demonstrated that the expression of $\beta 1$ - and $\beta 3$-integrin was not increased in the proximal aorta, where the expression levels of FAK and PYK2 were increased. Although the property of interaction between integrin and FAK in cytoplasm has not been well documented (Giancotti and Ruoslahti 1999), it is recognized that integrins have three functional states, including latent, active and ligand-occupied states (Luque et al. 1996) and that overexpression of FAK leads to phosphorylation of MAP kinases through activating integrins (Schlaepfer and Hunter 1997). Thus, activation of integrins might be involved in phosphorylation of tyrosine-kinases in hypertension. This point remains to be examined in future studies. Second, although we performed the in vivo experiment to evaluate the total and phosphorylated FAK and PYK2, we did not evaluate the intracellular mechanisms by mechanical stretch or pressure overloaded. This point also remains to be examined in future studies.

\section{Conclusions}

In the present study, we are able to demonstrate that phosphorylation of FAK and PYK2 is enhanced by elevated blood pressure but not by humoral factors in the rat aorta, demonstrating novel aspects of atherogenesis in hypertension.

\section{Acknowledgments}

This work was supported in part by the grants-in-aid (Nos. 15256003, 16209027, 16659192) from the Japanese Ministry of Education, Culture, Sports, Science and Technology, Tokyo, Japan.

\section{References}

Ali, M.H. \& Schumacker, P.T. (2002) Endothelial responses to mechanical stress: where is the mechanosensor? Crit. Care Med., 30, S198-206.

Avraham, H., Park, S.Y., Schinkmann, K. \& Avraham, S. (2000) RAFTK/Pyk2-mediated cellular signalling. Cell. Signal., 12, 123-133.

Bezie, Y., Lamaziere, J.M., Laurent, S., Challande, P., Cunha, R.S., Bonnet, J. \& Lacolley, P. (1998) Fibronectin expression and aortic wall elastic modulus in spontaneously hypertensive rats. Arterioscler. Thromb. Vasc. Biol., 18, 1027-1034.

Burridge, K., Turner, C.E. \& Romer, L.H. (1992) Tyrosine phosphorylation of paxillin and pp125FAK accompanies cell adhesion to extracellular matrix: a role in cytoskeletal assembly. $J$. Cell Biol., 119, 893-903.

Cai, X., Lietha, D., Ceccarelli, D.F., Karginov, A.V., Rajfur, Z., Jacobson, K., Hahn, K.M., Eck, M.J. \& Schaller, M.D. (2008) Spatial and temporal regulation of focal adhesion kinase activity in living cells. Mol. Cell. Biol., 28, 201-214.

Chan, P.C., Lai, J.F., Cheng, C.H., Tang, M.J., Chiu, C.C. \& Chen, H.C. (1999) Suppression of ultraviolet irradiation-induced apoptosis by overexpression of focal adhesion kinase in Madin-Darby canine kidney cells. J. Biol. Chem., 274, 2690126906.

Do e, Z., Fukumoto, Y., Takaki, A., Tawara, S., Ohashi, J., Nakano, M., Tada, T., Saji, K., Sugimura, K., Fujita, H., Hoshikawa, Y., Nawata, J., Kondo, T. \& Shimokawa, H. (2009) Evidence for Rho-kinase activation in patients with pulmonary arterial hypertension. Circ. J., 73, 1731-1739.

Fukumoto, Y., Deguchi, J.O., Libby, P., Rabkin-Aikawa, E., Sakata, Y., Chin, M.T., Hill, C.C., Lawler, P.R., Varo, N., Schoen, F.J., Krane, S.M. \& Aikawa, M. (2004) Genetically determined resistance to collagenase action augments interstitial collagen accumulation in atherosclerotic plaques. Circulation, 110, 1953-1959.

Fukumoto, Y., Shimokawa, H., Ito, A., Kadokami, T., Yonemitsu, Y., Aikawa, M., Owada, M.K., Egashira, K., Sueishi, K., Nagai, R., Yazaki, Y. \& Takeshita, A. (1997) Inflammatory cytokines cause coronary arteriosclerosis-like changes and alterations in the smooth-muscle phenotypes in pigs. J. Cardiovasc. Pharmacol., 29, 222-231.

Fukumoto, Y., Shimokawa, H., Kozai, T., Kadokami, T., Kuwata, K., Owada, M.K., Shiraishi, T., Kuga, T., Egashira, K. \& Takeshita, A. (1996) Tyrosine kinase inhibitor suppresses the (re) stenotic changes of the coronary artery after balloon injury in pigs. Cardiovasc. Res., 32, 1131-1140.

Fukumoto, Y., Tawara, S. \& Shimokawa, H. (2007) Recent progress in the treatment of pulmonary arterial hypertension: expectation for rho-kinase inhibitors. Tohoku J. Exp. Med., 211, 309-320.

Giancotti, F.G. \& Ruoslahti, E. (1999) Integrin signaling. Science, 285, 1028-1032.

Govindarajan, G., Eble, D.M., Lucchesi, P.A. \& Samarel, A.M. (2000) Focal adhesion kinase is involved in angiotensin IImediated protein synthesis in cultured vascular smooth muscle cells. Circ. Res., 87, 710-716.

Iwasaki, H., Yoshimoto, T., Sugiyama, T. \& Hirata, Y. (2003) Activation of cell adhesion kinase beta by mechanical stretch in vascular smooth muscle cells. Endocrinology, 144, 23042310.

Lee, R.M., Forrest, J.B., Garfield, R.E. \& Daniel, E.E. (1983) Comparison of blood vessel wall dimensions in normotensive hypertensive rats by histometric and morphometric methods. Blood Vessels, 20, 245-254.

Lehoux, S. \& Tedgui, A. (1998) Signal transduction of mechanical stresses in the vascular wall. Hypertension, 32, 338-345.

Li, C. \& Xu, Q. (2000) Mechanical stress-initiated signal transductions in vascular smooth muscle cells. Cell. Signal., 12, 435- 
445.

Li, X. \& Earp, H.S. (1997) Paxillin is tyrosine-phosphorylated by and preferentially associates with the calcium-dependent tyrosine kinase in rat liver epithelial cells. J. Biol. Chem., 272, 14341-14348.

Libby, P. \& Aikawa, M. (2002) Stabilization of atherosclerotic plaques: new mechanisms and clinical targets. Nat. Med., 8, $1257-1262$.

Luque, A., Gomez, M., Puzon, W., Takada, Y., Sanchez-Madrid, F. \& Cabanas, C. (1996) Activated conformations of very late activation integrins detected by a group of antibodies (HUTS) specific for a novel regulatory region (355-425) of the common beta 1 chain. J. Biol. Chem., 271, 11067-11075.

Nakano, M., Satoh, K., Fukumoto, Y., Ito, Y., Kagaya, Y., Ishii, N., Sugamura, K. \& Shimokawa, H. (2007) Important role of erythropoietin receptor to promote VEGF expression and angiogenesis in peripheral ischemia in mice. Circ. Res., 100, 662-669.

Ostergaard, H.L. \& Lysechko, T.L. (2005) Focal adhesion kinaserelated protein tyrosine kinase Pyk2 in T-cell activation and function. Immunol. Res., 31, 267-282.

Ramjaun, A.R. \& Hodivala-Dilke, K. (2009) The role of cell adhesion pathways in angiogenesis. Int. J. Biochem. Cell Biol., 41, 521-530.

Rice, D.C., Dobrian, A.D., Schriver, S.D. \& Prewitt, R.L. (2002) Src autophosphorylation is an early event in pressure-mediated signaling pathways in isolated resistance arteries. Hypertension, 39, 502-507.

Rocic, P., Griffin, T.M., McRae, C.N. \& Lucchesi, P.A. (2002) Altered PYK2 phosphorylation by ANG II in hypertensive vascular smooth muscle. Am. J. Physiol. Heart Circ. Physiol., 282, H457-465.

Ross, R. (1999) Atherosclerosis-an inflammatory disease. $N$. Engl. J. Med., 340, 115-126.

Saji, K., Fukumoto, Y., Suzuki, J., Fukui, S., Nawata, J. \& Shimokawa, H. (2007) Colchicine, a microtubule depolymerizing agent, inhibits myocardial apoptosis in rats. Tohoku J. Exp. Med., 213, 139-148.

Schlaepfer, D.D., Broome, M.A. \& Hunter, T. (1997) Fibronectinstimulated signaling from a focal adhesion kinase-c-Src complex: involvement of the Grb2, p130cas, and Nck adaptor proteins. Mol. Cell. Biol., 17, 1702-1713.

Schlaepfer, D.D., Hauck, C.R. \& Sieg, D.J. (1999) Signaling through focal adhesion kinase. Prog. Biophys. Mol. Biol., 71, 435-478.
Schlaepfer, D.D. \& Hunter, T. (1997) Focal adhesion kinase overexpression enhances ras-dependent integrin signaling to ERK2/mitogen-activated protein kinase through interactions with and activation of c-Src. J. Biol. Chem., 272, 1318913195.

Schwartz, S.M., Campbell, G.R. \& Campbell, J.H. (1986) Replication of smooth muscle cells in vascular disease. Circ. Res., 58, 427-444.

Shimokawa, H. (2000) Cellular and molecular mechanisms of coronary artery spasm: lessons from animal models. Jpn. Circ. J., 64, 1-12.

Shimokawa, H. (2002) Rho-kinase as a novel therapeutic target in treatment of cardiovascular diseases. J. Cardiovasc. Pharmacol., 39, 319-327.

Shyy, J.Y. \& Chien, S. (2002) Role of integrins in endothelial mechanosensing of shear stress. Circ. Res., 91, 769-775.

Sieg, D.J., Ilic, D., Jones, K.C., Damsky, C.H., Hunter, T. \& Schlaepfer, D.D. (1998) Pyk2 and Src-family protein-tyrosine kinases compensate for the loss of FAK in fibronectin-stimulated signaling events but Pyk2 does not fully function to enhance FAK-cell migration. EMBO J., 17, 5933-5947.

Smith, P.G., Garcia, R. \& Kogerman, L. (1998) Mechanical strain increases protein tyrosine phosphorylation in airway smooth muscle cells. Exp. Cell Res., 239, 353-360.

Tamura, K., Nyui, N., Tamura, N., Fujita, T., Kihara, M., Toya, Y., Takasaki, I., Takagi, N., Ishii, M., Oda, K., Horiuchi, M. \& Umemura, S. (1998) Mechanism of angiotensin II-mediated regulation of fibronectin gene in rat vascular smooth muscle cells. J. Biol. Chem., 273, 26487-26496.

Torsoni, A.S., Constancio, S.S., Nadruz, W. Jr., Hanks, S.K. \& Franchini, K.G. (2003) Focal adhesion kinase is activated and mediates the early hypertrophic response to stretch in cardiac myocytes. Circ. Res., 93, 140-147.

Vadali, K., Cai, X. \& Schaller, M.D. (2007) Focal adhesion kinase: an essential kinase in the regulation of cardiovascular functions. IUBMB Life, 59, 709-716.

Wang, H., Nawatam, J., Kakudo, N., Sugimura, K., Suzuki, J., Sakuma, M., Ikeda, J. \& Shirato, K. (2004) The upregulation of ICAM-1 and P-selectin requires high blood pressure but not circulating renin-angiotensin system in vivo. J. Hypertens., 22, 1323-1332.

Xiong, W. \& Parsons, J.T. (1997) Induction of apoptosis after expression of PYK2, a tyrosine kinase structurally related to focal adhesion kinase. J. Cell Biol., 139, 529-539. 